\title{
Synergistic Increase of BDNF Release from Rat Primary Cortical Neuron by Combination of Several Medicinal Plant-Derived Compounds
}

\author{
Se Jin Jeon ${ }^{1}$, Haerang BaK ${ }^{1}$, Jungeun SeO ${ }^{1}$, Kyung Ja Kwon ${ }^{2,3}$, Young Sun KAnG ${ }^{2}$, Hee Jin KIm \\ Jae Hoon CHEONG ${ }^{4}$, Jong Hoon RYu ${ }^{5}$, Kwang Ho Ko ${ }^{1}$, and Chan Young $\mathrm{SHIN}^{2,3, *}$ \\ ${ }^{1}$ Department of Pharmacology, College of Pharmacy and Research Institute of Pharmaceutical Sciences, Seoul National University, \\ Seoul 151-742, ${ }^{2}$ Institute for Biomedical Sciences and Technology and Institute of Functional Genomics, Konkuk University, \\ ${ }^{3}$ Department of Pharmacology and Research Institute of Medical Sciences, School of Medicine, Konkuk University, Seoul 143-701, \\ ${ }^{4}$ Department of Pharmacy, Sahmyook University, Seoul 139-742, ${ }^{5}$ Department of Oriental Pharmaceutical Science, College of Pharmacy, \\ Kyung Hee University, Seoul 130-701, Republic of Korea
}

(Received November 3, 2009; Revised January 10, 2010; Accepted January 11, 2010)

\begin{abstract}
Brain-derived neurotrophic factor (BDNF) is a neurotrophic factor involved in neuronal differentiation, plasticity, survival and regeneration. BDNF draws massive attention mainly due to the potential as a therapeutic target in neurological diseases such as depression and Alzheimer's disease. In a primary screening for the natural compounds enhancing BDNF release from cultured rat primary cortical neuron, we found that compounds such as baicalein, tanshinone lla, cinnamic acid, epiberberine, genistein and wogonin among many others increased BDNF release. All the compounds at $0.1 \mu \mathrm{M}$ of concentration barely showed stimulatory effect on BDNF induction, however, their combination (mixture 1; baicalein, tanshinone lla and cinnamic acid, mixture 2; epiberberine, genistein and wogonin) showed synergistic increase in BDNF release as well as mRNA and protein expression. The level of BDNF expression was comparable to the maximum BDNF stimulation attainable by a positive control oroxylin $A(20 \mu \mathrm{M})$ without cell toxicity as determined by MTT analysis. Both mixtures synergistically increased the phosphorylation of extracellular signal-regulated kinase (ERK) as well as CAMP response element binding protein (CREB), an immediate and essential regulator of BDNF expression. Similar to these results, mixture of these compounds synergistically inhibited the up-regulation of inducible nitric oxide synthase (iNOS) induced by lipopolysaccharide treatments in rat primary astrocytes. These results suggest that the combinatorial treatment of natural compounds in lower concentration might be a useful strategy to obtain sufficient BDNF stimulation in neurological disease condition such as depression, while minimizing potential side effects and toxicity of higher concentration of a single compound.
\end{abstract}

Keywords: BDNF, Cortical neuron, CREB, ERK, Synergism

\section{INTRODUCTION}

Brain-derived neurotrophic factor (BDNF) is a growth factor belongs to neurotrophin family. BDNF plays essential roles in neuronal survival and control of CNS development (Bibel and Barde, 2000). BDNF initiates cellular response by specific binding to tropomycin receptor kinase $B$ (TrkB) (Soppet et al., 1991).

Several researchers have reported that BDNF regulates

${ }^{*}$ Corresponding author

Tel: +82-2-2030-7834 Fax: +82-2-2049-6192

E-mail: chanyshin@kku.ac.kr neuronal survival (Sairanen et al., 2005), neuronal differentiation and synaptic plasticity (Huang and Reichardt, 2001), which make it important to understand the neurobiology of BDNF in CNS diseases including epilepsy ( $\mathrm{Hu}$ and Russek, 2008), depression (Ozan et al., 2009), obsessive-compulsive disorder (Hemmings et al., 2008) and bipolar disorder (Angelucci et al., 2005). Therefore, regulating BDNF levels in several neurological diseases would be an effective target for treatments. In facts, several types of anti-depressant reversed the decreased BDNF production in depressive patients (Duman, 2002).

One of the key regulators of BDNF expression is cyclic AMP response element binding protein (CREB). CREB is 
a nuclear transcription factor, which binds to promoter regions of target DNAs, which is called cyclic AMP response element sequence (CRE), as a homodimer or heterodimer (Maekawa et al., 1989). The activation of CREB is achieved by the regulation of phosphorylation by mitogen-activated protein kinase (MAPK, Ha and Redmond, 2008), cAMP- protein kinase A (PKA, Wang et al., 2006), calcium/calmodulin-dependent protein kinase II/IV (CaMKII/ IV, Sato et al., 2006), and phospholipase C (PLC) - protein kinase C (PKC, Cramer et al., 2008).

We recently screened several compounds from medicinal herbs, which have been known to possess neuroprotective effects and found that compounds like baicalein, tanshinone Ila, cinnamic acid, epiberberine, genistein and wogonin increased the production of BDNF from cultured rat primary neuron.

Baicalein and wogonin are flavonoid compounds from the rhizome of medicinal plants such as Scutellaria baicalensis. These compounds increase cognitive function (Liu et al., 2007) and inhibit the induction of pro-inflammatory cytokines (Chen et al., 2009). Tanshinone Ila is one of the main ingredients of the roots of Salvia miltiorrhiza with diterpenoid structure. It has been suggested that tanshinone Ila has neuroprotective effects in the animal models of ischemic stroke (Yu et al., 2007). Cinnamic acid is from the barks of Cinnamomum cassia and possesses core structure of aromatic unsaturated carboxylic acid with neuroprotective effects (Yamashita et al., 2007). Epiberberine is a major component of Coptis chinensis and has been suggested to have beneficial effects in Alzheimer's disease (Jung et al., 2009). Genistein can be extracted from several species of plants including Pueraria lobata and possesses flavonoid structure.

Recent reports from several researchers including us suggest that natural compounds from several different species including Angelica gigas and Cassia obtusifolia exert neuroprotective effects at least in part by the up-regulation of BDNF through the regulation of CREB phosphorylation (Cui et al., 2009). In this study, we tried to examine whether the combination of low concentration of natural compounds has synergistic effects on BDNF expression. The synergistic combinatorial effects of these natural products on BDNF production may provide new approaches to achieve the up-regulation of BDNF while minimizing cellular toxicity or adverse effects of individual compounds.

\section{MATERIALS AND METHODS}

\section{Materials}

Dulbecco's modified eagle medium (DMEM)/F12, neu- robasal medium and fetal bovine serum were purchased from GIBCO BRL (Grand Island, NY, USA). Trypsin and MTT [3-(4,5-dimethylthiazol-2-yl)-2-,5-diphenyltetrazolium bromide] were purchased from Sigma (St. Louis, MO, USA). Specific BDNF, CREB, phospho-CREB, ERK1/2, phospho-ERK1/2 antibodies were obtained from Cell signaling Technology (Beverly, MA, USA). An antibody against iNOS was purchased from Abcam (Cambridge, UK) and $\beta$-actin antibody was obtained from Sigma (St. Louis, MO, USA). The BDNF ELISA determination kit was from Promega (Madison, $\mathrm{WI}$ ) and all other reagents were provided by Sigma.

\section{Cell culture}

Cultured cells were prepared from the cerebral cortex of fetal Sprague Dawley (SD) rat at embryonic day 16 as previously described (Park et al., 2009). Briefly, cortices were dissected and digested with trypsin-ethylenediaminetetraacetic acid (EDTA) solution followed by dissociation with a Pasteur pipette. The dissociated cells were resuspended in neurobasal medium (NBM) containing B-27 supplements and plated onto poly-D-lysine-coated culture dishes. The media were half-replaced with fresh media every 3 days. Cultured cortical neurons were kept for 10-14 days at $37^{\circ} \mathrm{C}$ and $10 \% \mathrm{CO}_{2}$ incubator before experiments.

\section{Cell viability}

Cell viability after treatment of the chemicals was determined by MTT assay. After treatment, cells were treated with the MTT solution (final concentration, $5 \mu \mathrm{g} / \mathrm{ml}$ ) for $2 \mathrm{~h}$. The dark blue formazan crystals formed in intact cells were solubilized with lysis buffer $(20 \%$ sodium dodecylsulfate in $50 \%$ aqueous $N, N$-dimethylformamide). The absorbance of the sample was read at $540-595 \mathrm{~nm}$ with a microplate reader (Molecular Devices, Sunnylvale, CA, USA). Results were expressed as the percentage (\%) of MTT reduction, assuming that the absorbance of control cells was $100 \%$.

\section{Enzyme linked immunosorbent assay (ELISA)}

To measure the BDNF release into the culture spent medium, we used BDNF Emax immunoassay kit (Promega) according to the manufacturer's instruction. First, we coated 96-well plate with anti-BDNF mAb and incubated it overnight at $4^{\circ} \mathrm{C}$. The plates were washed twice with PBS-Tween and added $200 \mu$ of 1 X block \& sample buffer and then incubated at room temperature for additional 1 hour. After incubation, the plate was washed twice and added $200 \mu \mathrm{l}$ of the culture supernatants taken from the cultured cells. And then, the plates were incubated overnight at $4^{\circ} \mathrm{C}$ with continuous shaking. The next day, af- 
ter another washing, the anti-human BDNF Ab were added and incubated $2 \mathrm{~h}$. After incubation, the plates were washed twice and anti-IgY HRP were added (light-protected, RT incubation with shaking for $1 \mathrm{~h}$ ). The reaction was developed with tetramethylbenzidine developer solution and the absorbance was read at $450 \mathrm{~nm}$ after stopping the reaction with $1 \mathrm{~N} \mathrm{HCl}$. The value of sample absorbance was quantitatively analyzed using BDNF standard curve.

\section{Reverse transcription-polymerase chain reaction (RT- PCR)}

Total RNA was extracted from the harvested cells using the Trizol $^{\mathbb{R}}$ (Invitrogen, Carlsbad, CA, USA) reagent and 1 $\mu \mathrm{g}$ of total RNA was converted to cDNA by Superscript II reverse transcriptase (Invitrogen) at $42^{\circ} \mathrm{C}$ for 90 min using first-strand cDNA synthesis kit. The PCR amplification was performed using Maxime PCR premix kit (iNtRon, Seongnam, Korea) and was consisted of 30 cycles $\left(94^{\circ} \mathrm{C}, 1 \mathrm{~min}\right.$; $60^{\circ} \mathrm{C}, 1 \mathrm{~min} ; 72^{\circ} \mathrm{C}, 1 \mathrm{~min}$ ) with the oligonucleotide primers for BDNF and glyceraldehyde 3-phosphate dehydrogenase (GAPDH). The sequence for PCR was as follows.

- BDNF Forward: 5'-ata gga gac cct ccg caa ct-3' Reverse: 5'-ctg cca tgc atg aaa cac tt-3'

- GAPDH Forward: 5'-tcc ctc aag att gtc agc aa-3' Reverse: 5'-aga tcc aca acg gat aca tt-3'

The PCR reaction was analyzed by $1 \%$ agarose gel electrophoresis and was stained with ethidium bromide.

\section{Western blot}

The activation of ERK1/2 and CREB was determined by Western blot using antibodies specific for phosphorylated, active forms of ERK1/2 or CREB. Cells were treated with various combination of test drugs in serum free DMEM and were lysed with $100 \mu$ l of $2 \times$ sample buffer ( $4 \%$ w/v SDS, $20 \%$ glycerol, $200 \mathrm{mM}$ DTT, $0.1 \mathrm{M}$ Tris- $\mathrm{HCl}, \mathrm{pH} 6.8$, and $0.02 \%$ bromophenol blue). The samples were fractionated by $10 \%$ SDS-PAGE and electrotransferred to nitrocellulose (NC) membrane. The NC membrane was blocked with $1 \mu \mathrm{g} / \mathrm{ml}$ polyvinyl alcohol and then incubated at room temperature for $2 \mathrm{~h}$ with monoclonal antibody (mAb) against pERK1/2 or pCREB (Cell Signaling Technology) which was diluted at $1: 3,000$ in $5 \%$ Blotto. After three 10 min washes with PBS containing $0.2 \%$ Tween-20 (PBS-T), the NC membranes were incubated with peroxidase-labeled goat anti-mouse or rabbit lgG at room temperature for $2 \mathrm{~h}$. After extensive washing with PBS-T, the membranes were developed by enhanced chemiluminescence (Amersham, Buckinghampshire, UK). As loading controls, Western blot was performed using antibodies against total ERK (Cell Signaling Technology), total CREB (Cell Signaling Technology) as well as $\beta$-actin in $1: 30,000$ dilution (Sigma). The quantification of the band intensity in Western blot was performed by Image J software (1.42 ver.)

\section{Determination of Nitrite production}

After treatment, $100 \mu \mathrm{l}$ of medium from 24 well plates was mixed with the equal volume of Griess reagent $(0.1 \%$ naphthylethylenediamine dihydrochloride and $1 \%$ sulfanilamide in 5\% phosphoric acid) in 96 well plates at RT. After $10 \mathrm{~min}$, the absorbance at $540 \mathrm{~nm}$ was measured using an UV spectrophotometer (Spectra Fluor, Tecan, Austria).

\section{Statistical analysis}

Data were expressed as the means \pm S.E.M. and analyzed for statistical significance by using one-way analysis of variance (ANOVA), followed by Newman-Keuls test as a post hoc test. The criteria for statistical significance was $p$ $<0.01$.

\section{RESULTS}

We first determined the effects of various natural compounds used in this study on the BDNF production as well as cell viability. We treated rat primary cortical neuron with various concentrations of the test compounds for $24 \mathrm{~h}$. A flavonoid compound, oroxylin $\mathrm{A}(20 \mu \mathrm{M})$ has been reported

Table I. Effects of natural compounds on cell viability. Various concentrations of natural compounds or mixture of $0.1 \mu \mathrm{M}$ of each compound were added to primary rat cortical neurons and the viability of the cells was determined by MTT analysis. MTT signal was expressed as $\%$ of control. Data represent mean \pm S.E.M $(n=3)$

\begin{tabular}{|c|c|c|c|c|}
\hline & Con & $0.1 \mu \mathrm{M}$ & $0.3 \mu \mathrm{M}$ & $1.0 \mu \mathrm{M}$ \\
\hline Baicalein (Bai) & $100.00 \pm 1.79$ & $103.61 \pm 1.53$ & $97.23 \pm 0.46$ & $102.94 \pm 2.56$ \\
\hline Tanshinone Ila (Tan) & $100.00 \pm 1.58$ & $98.53 \pm 1.69$ & $98.94 \pm 3.43$ & $91.96 \pm 4.84$ \\
\hline Cinnamic acid (Cin) & $100.00 \pm 1.65$ & $91.92 \pm 3.13$ & $93.57 \pm 1.42$ & $89.21 \pm 3.76$ \\
\hline Mixture 1 (Bai+Tan+Cin) & $100.00 \pm 2.37$ & - & $90.73 \pm 3.11$ & - \\
\hline Epiberberine (Epi) & $100.00 \pm 1.90$ & $106.34 \pm 3.68$ & $104.20 \pm 3.62$ & $106.37 \pm 1.59$ \\
\hline Genistein (Gen) & $100.00 \pm 4.45$ & $98.46 \pm 4.24$ & $114.70 \pm 10.71$ & $117.01 \pm 1.66$ \\
\hline Wogonin (Wog) & $100.00 \pm 2.38$ & $96.79 \pm 2.07$ & $92.83 \pm 1.43$ & $98.47 \pm 1.54$ \\
\hline Mixture 2 (Epi+Gen+Wog) & $100.00 \pm 2.29$ & - & $107.73 \pm 3.96$ & - \\
\hline
\end{tabular}


to increase BDNF production and CREB phosphorylation in rat brain (Kim et al., 2006) and therefore used as a positive control throughout this study. All the compounds used in this study, i.e. baicalein, tanshinone Ila, cinnamic acid, epiberberine, genistein, and wogonin did not show significant neuronal cytotoxicity, which was determined by MTT assay, at concentrations of $0.1,0.3$, and $1.0 \mu \mathrm{M}$. To investigate the synergistic effect of these compounds, we mixed three of each compounds at $0.1 \mu \mathrm{M}$ concentration and in this condition, all the mixtures did not show significant effects on cell viability (Table I). Oroxylin A (20 $\mu \mathrm{M})$, used as a positive control, also did not produce significant cytotoxicity $(95.2 \pm 3.3 \%$ as compared with control).

Rat primary cortical neurons were treated with various concentrations of natural compounds for $24 \mathrm{~h}$ and the culture supernatants were analyzed for BDNF release by ELISA assay. All six compounds showed modest increase in BDNF release at $1 \mu \mathrm{M}$ concentration, while $20 \mu \mathrm{M}$ of oroxylin $A$, which has been used as a positive control, showed a significant increase in BDNF release (Fig. 1A). We mixed $0.1 \mu \mathrm{M}$ of baicalein, tanshinone lla and cinnamic acid together (mixture 1), and mixed epiberberine, genistein, and wogonin together (mixture 2). Although $0.3 \mu \mathrm{M}$ of each compound (shown here are cinnamic acid, and epiberberine) showed only marginal effects on BDNF production, if any, both mixtures of $0.1 \mu \mathrm{M}$ compounds significantly increased BDNF release (Fig. 1B). These results suggest that co-treatment of natural compounds used in this study has synergistic effects on BDNF release.
One of the key regulators of BDNF production is the activation of CREB, which might be regulated by ERK activation as well as PKA and calcium signaling. We investigated the activation of CREB and ERK pathway by our compounds using Western blot against phospho-specific CREB as well as phospho-specific ERK. Compared to the representative compounds of mixtures 1 (cinnamic acid) and 2 (epiberberine), both mixtures showed significantly higher phospho-ERK level (Fig. 2A). Likewise, the level of phopho-CREB was increased about 2-fold compared to each single compound (Fig. 2B). Interestingly, mixture 2 increased ERK activity compared to single compound by $130 \%$ but increased $\mathrm{p}$-CREB activity by $200 \%$, suggesting that the compounds used in mixture 2 may have additional effects on CREB-regulating pathways which is not related to ERK pathways. To check the role of ERK pathway on BDNF production, we pretreated the cells with a MAPK pathway inhibitor U0126, and it reduced the stimulated BDNF induction by mixtures (Fig. 2C).

Next, we investigated the expression of BDNF by RT-PCR analysis. The expression level of BDNF mRNA was significantly higher in rat primary cortical neurons treated with mixtures 1 and 2 compared with each representative single compound (Fig. 3A). Consistent with these results the level of BDNF protein, which was determined by Western blot using an antibody specific against BDNF, was significantly increased by the treatment of mixtures 1 and 2 (Fig. 3B). These results suggest that the increase in $\mathrm{BDNF}$ release is mainly regulated at the level of transcriptional control. Altogether, these results suggest that natu-

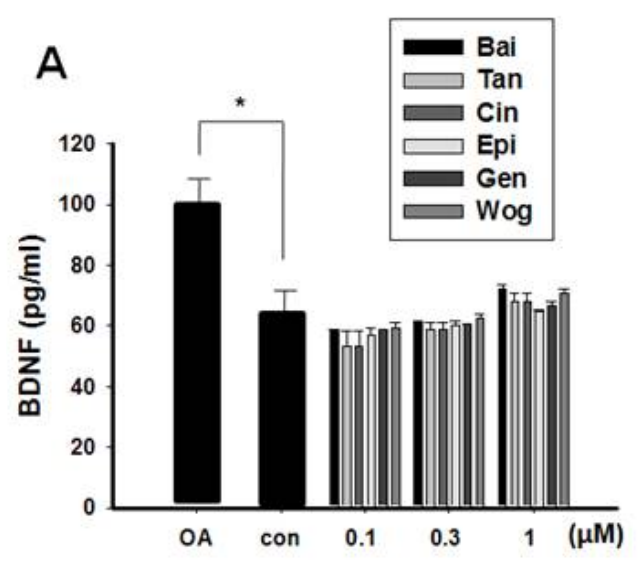

B

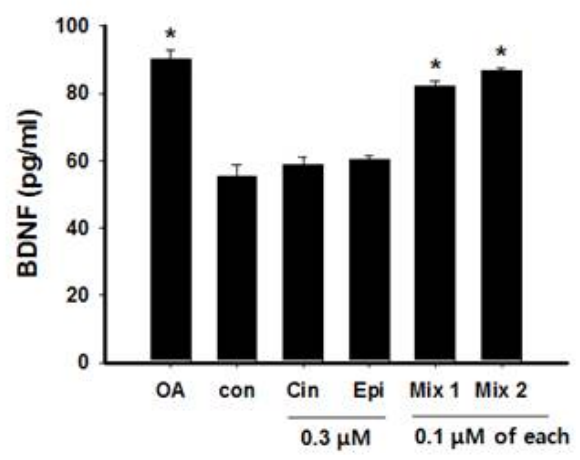

Fig. 1. The effects of natural compounds on BDNF release from rat primary cortical neurons. (A) Indicated concentrations of each compound or $20 \mu \mathrm{M}$ oroxylin A (a positive control) was added to the cells and the culture supernatants were analyzed for BDNF release using ELISA kit as described in Materials and Methods. Bai: baicalein, Tan: tanshinone Ila, Cin: cinnamic acid, Epi: epiberberine, Gen: genistein, Wog: wogonin. (B) Cinnamic acid (0.3 $\mu \mathrm{M}$ ) or epiberberine (Cin and Epi) or mixtures of $0.1 \mu \mathrm{M}$ of three of each compound (Mix 1 and Mix 2) was added as above and analyzed for BDNF release. Note the increased BDNF release as compared to $0.3 \mu \mathrm{M}$ of each compound alone. Data represent mean \pm S.E.M. * Significantly different as compared with control ( $p$ $<0.05, \mathrm{n}=5$ ). 
A
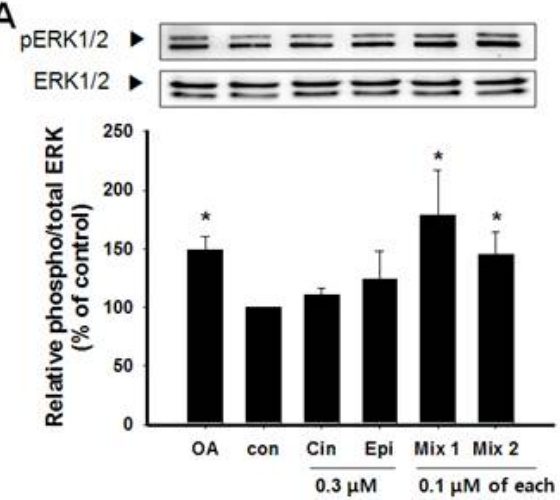

B
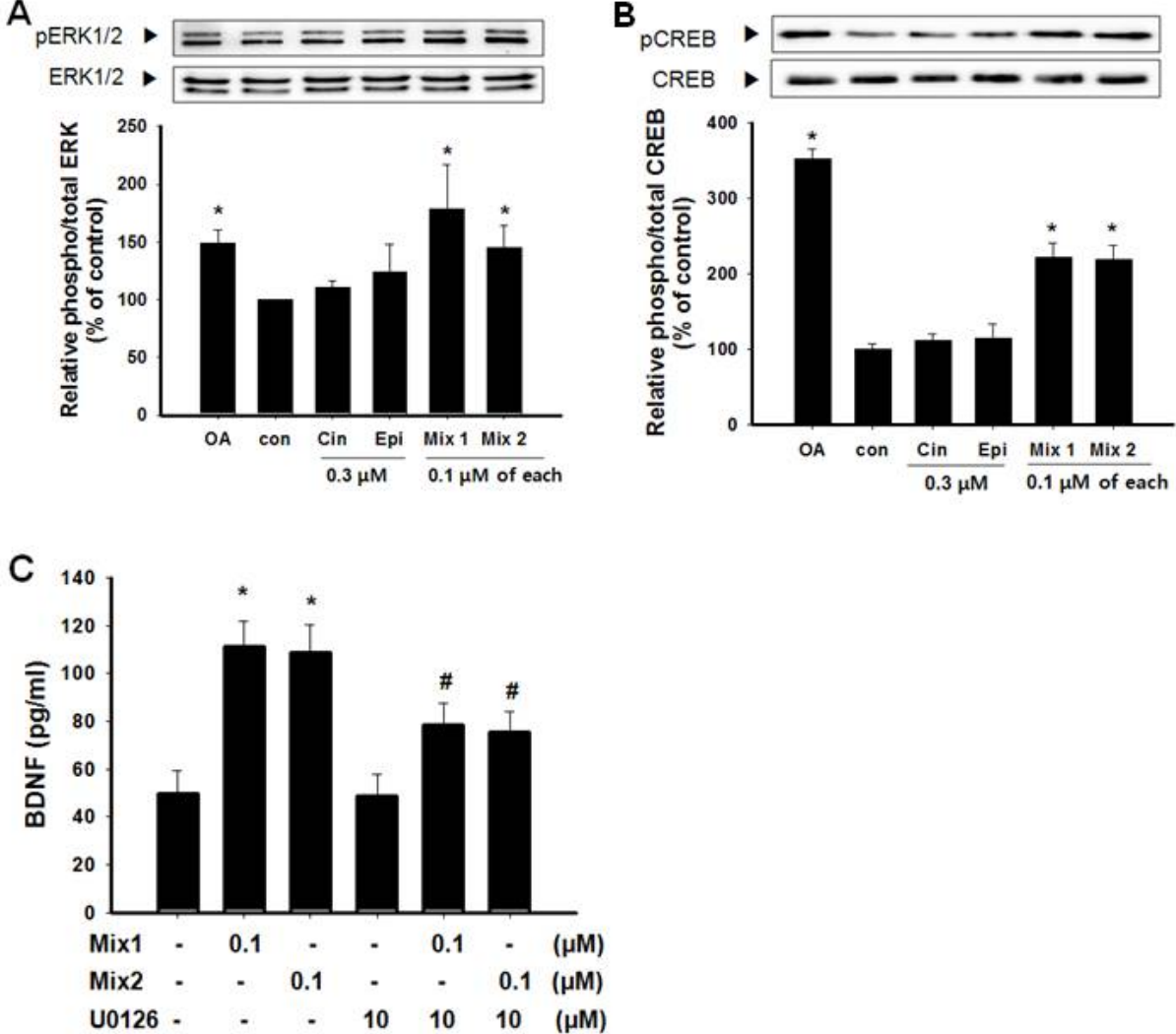

A
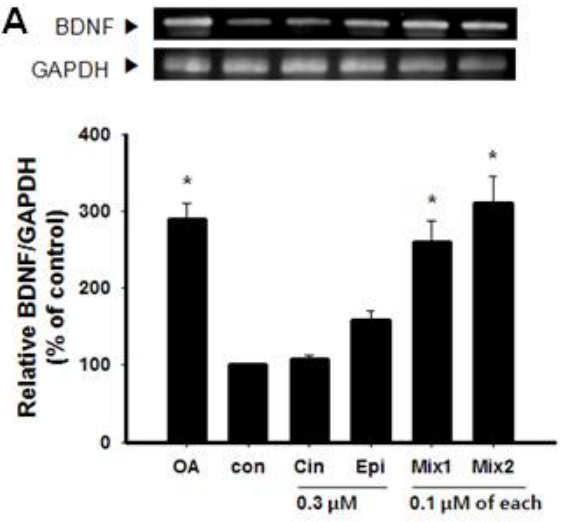

B
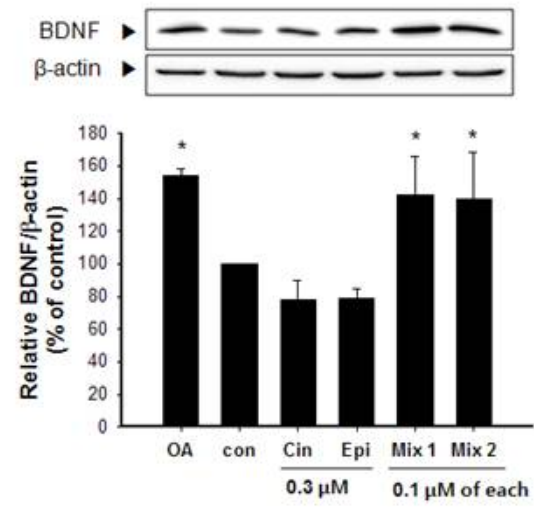

Fig. 2. The effects of natural compounds on ERK and CREB phosphorylation in rat primary cortical neurons. A positive control ( $20 \mu \mathrm{M}$ oroxyin A), $0.3 \mu \mathrm{M}$ cinnamic acid or epiberberine (Cin and Epi) or mixtures of $0.1 \mu \mathrm{M}$ of three of each compound (Mix 1 and Mix 2) was added as above and analyzed for (A) ERK phosphorylation or (B) CREB phosphorylation by Western blot using a specific antibody against phospho-ERK or phospho-CREB. Graphs represent quantification of Western blot band intensity. (C) The effects of an MAPK inhibitor U0126 $(10 \mu \mathrm{M})$ on natural compounds-induced BDNF production. Data represent mean \pm S.E.M. *Significantly different as compared with control $(p<0.05, n=5)$, "Significantly different as compared with mixture treated alone $(p<0.05, n=5)$.

Fig. 3. The effects of natural compounds on BDNF expression in rat primary cortical neurons. (A) RT-PCR of BDNF mRNA expression. $0.3 \mu \mathrm{M}$ cinnamic acid or epiberberine (Cin and Epi) or mixtures of $0.1 \mu \mathrm{M}$ of three of each compound (Mix 1 and Mix 2) was added as above and analyzed for BDNF mRNA expression by RT-PCR. Graph represents quantification of electrophoretic bands. (B) Western blot determination of BDNF expression. After treatment, cells were harvested and analyzed for the BDNF protein expression by Western blot. Graph represents quantification data of band intensity. Data represent mean \pm S.E.M. *Significantly different as compared with control $(p<0.05, \mathrm{n}=5)$. ral compounds used in this study have synergistic effects on BDNF expression and release by mechanism involving the synergistic activation of key intracellular signaling pathways such as ERK and CREB.

Since many natural products as well as six compounds used in our experiments had been known for anti-oxidative effects including inhibition of iNOS expression, we also examined whether the mixtures have synergistic inhibitory effect on LPS-stimulated iNOS production in rat primary astrocytes. We stimulated rat primary astrocytes with lipopolysaccharide (LPS, $0.2 \mu \mathrm{g} / \mathrm{ml}$ ), a well known astrocyte and microglial activator, to induce inducible nitric oxide 
A

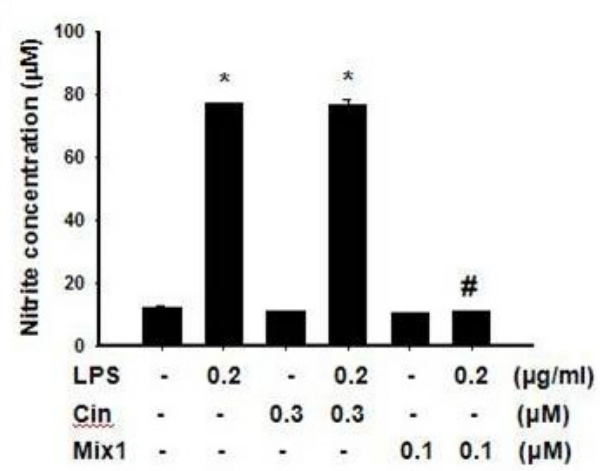

C

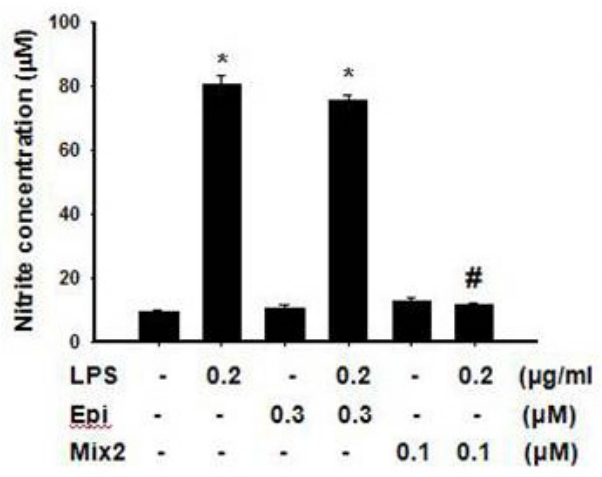

B
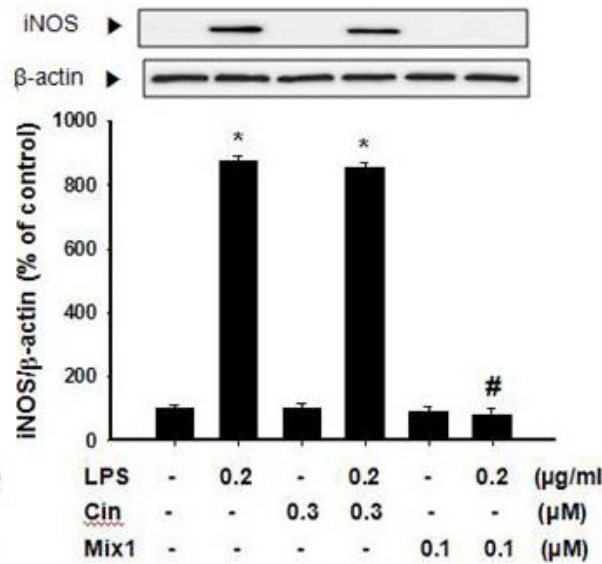

D
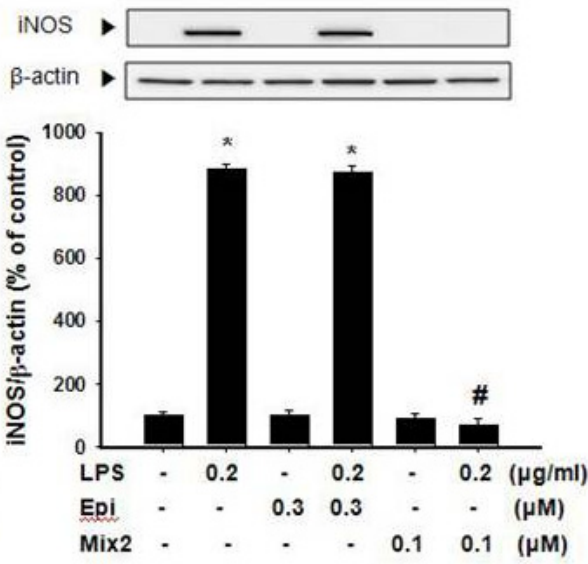

Fig. 4. The effects of natural compounds on nitrite release and iNOS over-production in LPS-stimulated rat primary astrocytes. Rat primary astrocytes were pretreated with $0.3 \mu \mathrm{M}$ cinnamic acid or epiberberine (Cin and Epi) or mixtures of $0.1 \mu \mathrm{M}$ of three of each compound (Mix 1 and Mix 2) and then stimulated with LPS. After $24 \mathrm{~h}$, cells were analyzed for nitrite production by Griess reaction ( $A$ and $C$ ) or iNOS expression by Western blot using a specific antibody against iNOS (B and D). Graph represents quantification of Western blot band intensity. Data represent mean \pm S.E.M. *Significantly different as compared with control $(p<0.05, \mathrm{n}=5)$, "Significantly different as compared with mixture treated alone $(p<0.05, \mathrm{n}=5)$. synthase (iNOS) over-expression. LPS, as reported previously, by itself or in combination with other cytokines including interferon- $\gamma$ induces iNOS production in rat glial cells (Bhat et al., 1998). The over-expression of iNOS and nitrite production was not inhibited by $0.3 \mu \mathrm{M}$ single compounds (cinnamic acid and epiberberine), however, pretreatment of the cells with mixture 1 or mixture 2 completely prevented the over-production of nitrite and iNOS (Fig. 4). These results suggest that the combination of these compounds also showed synergistic antioxidant effects in LPS-stimulated astrocytes.

\section{DISCUSSION}

BDNF is a member of neurotrophic factors involved in the regulation of neuronal survival (Sairanen et al., 2005), synaptic function, synaptic plasticity as well as neuronal differentiation (Huang and Reichardt, 2001). In addition, it is essential for the normal development of neuron (Ernfors et al., 1995) and provide beneficial effect in several neuro- logical disease states including depression (Duman et al., 1997), schizophrenia (Sasaki et al., 1997) as well as neurodegenerative diseases such as Huntington disease, Alzheimer disease and Parkinson disease (Zuccato and Cattaneo, 2009). Although originally discovered in brain and hence it gets the name BDNF, it is also found in PNS (Conner et al., 1997). In brain, BDNF is highly expressed in structures involved in learning and memory and higher executive functions (Hofer et al., 1990).

In our initial screening of natural compounds modulating BDNF release in rat primary cortical neuron, six compounds used in this study showed increased BDNF release at more than $1 \mu \mathrm{M}$ concentrations (data not shown). These compounds have been reported to have either neuroprotective or anti-oxidative effects (Kim and Kim, 2000; Piao et al., 2004; Xia et al., 2005; Jung et al., 2009; Mu et al., 2009; Yu et al., 2009). However, not all natural compounds having neuroprotective or anti-oxidative effects showed increased BDNF release. For example, a flavonoid or a lignan compound such as benzoylpaeniflorin 
and phylligenin, which have relatively strong anti-inflammatory and anti-oxidative effects, did not increase BDNF release (data not shown) suggesting simple anti-oxidative properties are not sufficient for the increased BDNF release. Consistent with this speculation, we did not find any common structural determinants to mediate BDNF release in the six compounds tested in this study.

Considering the structural diversities of the six compounds tested in this study, it is reasonable assumption that these compounds regulate different subsets of receptors and intracellular signaling pathways, which mediates the phosphorylation of CREB, a common denominator of the increased BDNF production and release. Currently, four major pathways including CAMP-PKA, MAPKp90 ribosomal S6 kinase (RSK), $\mathrm{Ca}^{2+}$-CAMKII and PLC$\mathrm{PKC}$ have been suggested to modulate CREB phosphorylation and BDNF production. Interestingly, mixture 2 increased the phosphorylation of CREB as much as mixture 1 , however, the effects on the phosphorylation of ERK was weaker than that of mixture 1 , suggesting that the ingredients in mixture 2 (epiberberine, genistein, and wogonin) may have stimulatory effects on other pathways in addition to ERK pathway. Regarding the activation of ERK, it has been suggested that neurotrophic factors acting on TrkB including BDNF and NT-4 can activate ERK phosphorylation, thereby forming auto-regulatory feed-forward activating loop of BDNF expression (Rumajogee et al., 2002). It should be investigated in the future whether compounds used in this study, especially mixture 1 (baicalein, tanshinone Ila and cinnamic acid) may modulate the production of other growth factors as well.

Regarding the synergistic mechanism of combinatorial use of natural products, four major mechanisms have described (Wagner and Ulrich-Merzenich, 2009). First, the synergistic effects may involve activation of multiple targets. The second is pharmacokinetic effect, in which solubility, resorption rate or bioavailability is increased. Third, the interaction of compounds may lead to the resistance against bacterial degradation. Fourth and the last, the combination of compounds may produce neutralization or elimination of the toxic action or adverse effects of other compounds. In this study, the most plausible explanation of the observed synergistic effects is the cooperative activation of multiple targets by the constituents of the mixture. Drug targets include enzymes, substrates, metabolites, proteins, receptors, ion channels, transport proteins, DNA/ RNA, ribosome, monoclonal antibodies and still unknown mechanism (Imming et al., 2006). Although we do not know exactly what is the principal targets of the each compounds used in this study, it is obvious that if these mole- cules share a single target, we might expect simple additive effects or less than additive effects depending on the level of saturation of the target. The structural diversity of the molecules and several reports suggesting the activation of many different signaling pathways such as cAMPPKA (Wang et al., 2006), PLC-PKC (Cramer et al., 2008), ERK-RSK (Ha and Redmond, 2008) and $\mathrm{Ca}^{2+}$-CAMKII (Sato et al., 2006) by these compounds, which is essential for CREB phophorylation and BDNF production, additionally support the idea of synergistic action by activation of multiple targets.

Using mixtures of reagents as a therapeutic agents has several advantages over using a single compound in higher concentration (Wagner and Ulrich-Merzenich, 2009). First of all, we can use a significantly low dose of drugs to achieve similar pharmacological effects. While administration of $0.3 \mu \mathrm{M}$ of cinnamic acid or epiberberine showed only marginal increase in BDNF production, combining three compounds together at $0.1 \mu \mathrm{M}$ each concentration showed comparable increase in BDNF release to $20 \mu \mathrm{M}$ oroxylin A, a positive control. The smaller amount of drug with similar or superior activity means that less toxicity or adverse effects might be achieved with the combination of synergistic drugs. In ischemic stroke, it has been suggested that the combined use of a gamma-aminobutyric acid (GABA) agonist and a glutamate antagonist provides synergistic neuroprotective effect (Lyden et al., 2000). Similarly, the combined use of the drugs used in bipolar disorder such as valproic acid and lithium showed neuroprotective effects both in vivo and in vitro (Feng et al., 2008). Likewise, synergistic action of combined treatment of several neurotrophin family members together on spinal cord injury (Novikova et al., 2000) and combination of $\mathrm{N}$-methyl D-aspartate (NMDA) and $\alpha$-amino-3-hydroxyl5-methyl-4-isoxazole-propionate (AMPA) receptor antagonists on acute ischemic injury have been reported (Liu et al., 2009).

Taken together, the combinatorial treatment of several natural compounds to rat primary cortical neuron produced synergistic stimulatory effects on BDNF production and release. With the treatment of lower concentrations of each compound, while retaining similar pharmacological effects, this approach may provide one way to minimize cellular toxicity and adverse effects in the treatment of neurodegenerative diseases and psychological diseases such as depression. The next step will be the delineation of the specific signaling pathways involved in the regulation of BDNF production by each single compound and the molecular mechanism underlying the synergistic effects. 


\section{ACKNOWLEDGMENTS}

This work was supported in part by Institute for Biomedical Sciences and Technology, Konkuk University (C. Y. Shin, 2008) and also in part by a grant (08172KFDA261) funded by the Korean Food and Drug Administration (K. H. Ko).

\section{REFERENCES}

Angelucci, F., Brene, S. and Mathe, A. A. (2005). BDNF in schizophrenia, depression and corresponding animal models. Mol. Psychiatry 10, 345-352.

Bhat, N. R., Zhang P, Lee J. C. and Hogan E. L. (1998). Extracellular signal-regulated kinase and p38 subgroups of mitogen-activated protein kinases regulate inducible nitric oxide synthase and tumor necrosis factor-alpha gene expression in endotoxin-stimulated primary glial cultures. $J$ Neurosci. 18,1633-1641.

Bibel, M. and Barde, Y. A. (2000). Neurotrophins: key regulators of cell fate and cell shape in the vertebrate nervous system. Genes Dev. 14, 2919-2937.

Chen, S. O., Fang, S. H., Shih, D. Y., Chang, T. J. and Liu, J. J. (2009). Recombinant core proteins of Japanese encephalitis virus as activators of the innate immune response. Virus Genes 38, 10-18.

Conner, J. M., Lauterborn, J. C., Yan, Q., Gall, C. M. and Varon, S. (1997). Distribution of brain-derived neurotrophic factor (BDNF) protein and mRNA in the normal adult rat CNS: evidence for anterograde axonal transport. J. Neurosci. 17, 2295-2313.

Cramer, T., Juttner, S., Plath, T., Mergler, S., Seufferlein, T., Wang, T. C., Merchant, J. and Hocker, M. (2008). Gastrin transactivates the chromogranin A gene through MEK-1/ ERK- and PKC-dependent phosphorylation of Sp1 and CREB. Cell Signal 20, 60-72.

Cui, Q., Zhang, J., Zhang, L., Li, R. and Liu, H. (2009). Angelica injection improves functional recovery and motoneuron maintenance with increased expression of brain derived neurotrophic factor and nerve growth factor. Curr. Neurovasc. Res. 6, 117-123.

Duman, R. S. (2002). Pathophysiology of depression: the concept of synaptic plasticity. Eur. Psychiatry 17 Suppl 3, 306-310.

Duman, R. S., Heninger, G. R. and Nestler, E. J. (1997). A molecular and cellular theory of depression. Arch. Gen. Psychiatry 54, 597-606.

Ernfors, P., Van De Water, T., Loring, J. and Jaenisch, R. (1995). Complementary roles of BDNF and NT-3 in vestibular and auditory development. Neuron 14, 1153-1164.

Feng, H. L., Leng, Y., Ma, C. H., Zhang, J., Ren, M. and Chuang, D. M. (2008). Combined lithium and valproate treatment delays disease onset, reduces neurological deficits and prolongs survival in an amyotrophic lateral sclerosis mouse model. Neuroscience 155, 567-572.

$\mathrm{Ha}$, S. and Redmond, L. (2008). ERK mediates activity dependent neuronal complexity via sustained activity and CREB-mediated signaling. Dev. Neurobiol. 68, 1565-1579.
Hemmings, S. M., Kinnear, C. J., Van der Merwe, L., Lochner C., Corfield, V. A., Moolman-Smook, J. C. and Stein, D. J. (2008). Investigating the role of the brain-derived neurotrophic factor (BDNF) val66met variant in obsessive-compulsive disorder (OCD). World J. Biol. Psychiatry 9, 126-134.

Hofer, M., Pagliusi, S. R., Hohn, A., Leibrock, J. and Barde, Y. A. (1990). Regional distribution of brain-derived neurotrophic factor mRNA in the adult mouse brain. EMBO. J. 9, 24592464

Hu, Y. and Russek, S. J. (2008). BDNF and the diseased nervous system: a delicate balance between adaptive and pathological processes of gene regulation. J. Neurochem. 105, 1-17.

Huang, E. J. and Reichardt, L. F. (2001). Neurotrophins: roles in neuronal development and function. Annu. Rev. Neurosci. 24, 677-736.

Imming, P., Sinning, C. and Meyer, A. (2006). Drugs, their targets and the nature and number of drug targets. Nat. Rev. Drug Discov. 5, 821-834.

Jung, H. A., Min, B. S., Yokozawa, T., Lee, J. H., Kim, Y. S. and Choi, J. S. (2009). Anti-Alzheimer and antioxidant activities of Coptidis Rhizoma alkaloids. Biol. Pharm. Bull. 32 , 1433-1438.

Kim, D. H., Jeon, S. J., Son, K. H., Jung, J. W., Lee, S., Yoon, B. H., Choi, J. W., Cheong, J. H., Ko, K. H. and Ryu, J. H. (2006). Effect of the flavonoid, oroxylin A, on transient cerebral hypoperfusion-induced memory impairment in mice. Pharmacol. Biochem. Behav. 85, 658-668.

Kim, S. R. and Kim, Y. C. (2000). Neuroprotective phenylpropanoid esters of rhamnose isolated from roots of Scrophularia buergeriana. Phytochemistry 54, 503-509.

Liu, C., Lin, N., Wu, B. and Qiu, Y. (2009). Neuroprotective effect of memantine combined with topiramate in hypoxic-ischemic brain injury. Brain Res. 1282, 173-182.

Liu, C., Wu, J., Gu, J., Xiong, Z., Wang, F., Wang, J., Wang, W. and Chen, J. (2007). Baicalein improves cognitive deficits induced by chronic cerebral hypoperfusion in rats. Pharmacol. Biochem. Behav. 86, 423-430.

Lyden, P. D., Jackson-Friedman, C., Shin, C. and Hassid, S. (2000). Synergistic combinatorial stroke therapy: A quantal bioassay of a GABA agonist and a glutamate antagonist. Exp. Neurol. 163, 477-489.

Maekawa, T., Sakura, H., Kanei-Ishii, C., Sudo, T., Yoshimura T., Fujisawa, J., Yoshida, M. and Ishii, S. (1989). Leucine zipper structure of the protein CRE-BP1 binding to the cyclic AMP response element in brain. EMBO. J. 8, 2023-2028.

Mu, X., He, G., Cheng, Y., Li, X., Xu, B. and Du, G. (2009). Baicalein exerts neuroprotective effects in 6-hydroxydopamine-induced experimental parkinsonism in vivo and in vitro. Pharmacol. Biochem. Behav. 92, 642-648.

Novikova, L. N., Novikov, L. N. and Kellerth, J. O. (2000). BDNF abolishes the survival effect of NT-3 in axotomized Clarke neurons of adult rats. J. Comp. Neurol. 428, 671-680.

Ozan, E., Okur, H., Eker, C., Eker, O. D., Gonul, A. S. and Akarsu, N. (2010). The effect of depression, BDNF gene val66met polymorphism and gender on serum BDNF levels. Brain Res. Bull. 15, 61-65.

Park, J., Koito, H., Li, J. and Han, A. (2009). Microfluidic compartmentalized co-culture platform for CNS axon myelination research. Biomed. Microdevices 11, 1145-1153. 
Piao, H. Z., Jin, S. A., Chun, H. S., Lee, J. C. and Kim, W. K. (2004). Neuroprotective effect of wogonin: potential roles of inflammatory cytokines. Arch. Pharm. Res. 27, 930-936.

Rumajogee, P., Madeira, A., Verge, D., Hamon, M. and Miquel, M. C. (2002). Up-regulation of the neuronal serotoninergic phenotype in vitro: BDNF and cAMP share Trk B-dependent mechanisms. J. Neurochem. 83, 1525-1528.

Sairanen, M., Lucas, G., Ernfors, P., Castren, M. and Castren, E. (2005). Brain-derived neurotrophic factor and antidepressant drugs have different but coordinated effects on neuronal turnover, proliferation, and survival in the adult dentate gyrus. J. Neurosci. 25, 1089-1094.

Sasaki, T., Dai, X. Y., Kuwata, S., Fukuda, R., Kunugi, H., Hattori, M. and Nanko, S. (1997). Brain-derived neurotrophic factor gene and schizophrenia in Japanese subjects. Am. J. Med. Genet. 74, 443-444.

Sato, K., Suematsu, A., Nakashima, T., Takemoto-Kimura, S., Aoki, K., Morishita, Y., Asahara, H., Ohya, K., Yamaguchi, A., Takai, T. (2006). Regulation of osteoclast differentiation and function by the CaMK-CREB pathway. Nat. Med. 12 , 1410-1416.

Soppet, D., Escandon, E., Maragos, J., Middlemas, D. S., Reid S. W., Blair, J., Burton, L. E., Stanton, B. R., Kaplan, D. R., Hunter, T., et al. (1991). The neurotrophic factors brainderived neurotrophic factor and neurotrophin-3 are ligands for the trkB tyrosine kinase receptor. Cell 65, 895-903.

Wagner, H. and Ulrich-Merzenich, G. (2009). Synergy research: approaching a new generation of phytopharma- ceuticals. Phytomedicine 16, 97-110.

Wang, Z., Hu, S. Y., Lei, D. L. and Song, W. X. (2006). Effect of chronic stress on PKA and P-CREB expression in hippocampus of rats and the antagonism of antidepressors. Zhong Nan Da Xue Xue Bao Yi Xue Ban 31, 767-771.

Xia, W. J., Yang, M., Fok, T. F., Li, K., Chan, W. Y., Ng, P. C., Ng, H. K., Chik, K. W., Wang, C. C., Gu, G. J., et al. (2005). Partial neuroprotective effect of pretreatment with tanshinone IIA on neonatal hypoxia-ischemia brain damage. Pediatr. Res 58, 784-790.

Yamashita, K., Kotani, Y., Nakajima, Y., Shimazawa, M., Yoshimura, S., Nakashima, S., Iwama, T. and Hara, H. (2007). Fasudil, a Rho kinase (ROCK) inhibitor, protects against ischemic neuronal damage in vitro and in vivo by acting directly on neurons. Brain Res. 1154, 215-224.

Yu, H. L., Li, L., Zhang, X. H., Xiang, L., Zhang, J., Feng, J. F. and Xiao, R. (2009). Neuroprotective effects of genistein and folic acid on apoptosis of rat cultured cortical neurons induced by beta-amyloid 31-35. Br. J. Nutr. 102, 655-662.

Yu, X. Y., Lin, S. G., Zhou, Z. W., Chen, X., Liang, J., Liu, P. Q., Duan, W., Chowbay, B., Wen, J. Y., Li, C. G. and Zhou, S. F. (2007). Role of P-glycoprotein in the intestinal absorption of tanshinone IIA, a major active ingredient in the root of Salvia miltiorrhiza Bunge. Curr. Drug Metab. 8, 325-340.

Zuccato, C. and Cattaneo, E. (2009). Brain-derived neurotrophic factor in neurodegenerative diseases. Nat. Rev. Neurol. 5, 311-322. 